\title{
THE ARTERIES OF THE PANCREAS
}

\author{
RUSSELL T, WOODBURNE AND LLOYD L. OLSEN \\ Departments of Anatomy and Surgery, University of Michigan \\ Medical School, Ann Arbor
}

FIVE FIGURES

\section{INTRODUCTION}

The present study was undertaken in response to the very considerable inadequacy in the description of the pancreatic blood supply in anatomical textbooks. Especially is the surgeon currently concerned with the feasibility of surgical attack on the pancreas and the success of such operative procedures may depend in part on a knowledge of specific vessels reaching the gland. The authors were particularly interested in defining, if possible, a general pattern in the blood supply of this organ as well as in determining the range of variation of the vessels concerned.

\section{REVIEW OF LITERATLRE}

An exhaustive review of the literature concerning investigations of the blood vessels will not be attempted here. A complete and informative review of the studies up to 1929 is included in the report by Petrén ('29). Many studies oriented primarily toward the duodenum give only partial information concerning the arteries of the pancreas. Other studies suffer in that their statistical analysis is based on too few specimens. It will be profitable, however, to give emphasis here to certain studies which materially advanced our knowledge of the arteries of the pancreas.

The earliest description of certain important pancreatic arteries appears to have been that by Haller in 1742 who recognized double arterial arches or arcades anterior and 
posterior to the head of the pancreas and serving both duodenum and pancreas. His was the first description of the posterior superior pancreaticoduodenal branch of the gastroduodenal artery to the posterior arcade. Haller also described the A. pancreatica magna suprema, a vessel which has a very frequent occurrence but is perhaps more commonly recognized at present as the dorsal pancreatic artery. With the description of the A. transversa pancreatica as a continuation of the A. pancreatica magna suprema Haller very nearly rounded out a complete description of this important blood supply. Haller's priority of deseription on all points may be open to question, however, since Winslow described in 1732 a duodenal or intestinal branch of the gastroduodenal artery which "sometimes is double," and in speaking of the superior mesenteric artery described "a small branch near its origin which, dividing into two, goes to the lower side of the head of the pancreas and neighboring part of the duodenum, communicating with the intestinalis by small arches." These early studies remained essentially unrecognized for about 150 years when Wiart (1899), writing on the form and relations of the pancreas, gave a good description of the pancreaticoduodenal arcades. This included a description of a right superior pancreaticoduodenal artery as a proximal branch of the gastroduodenal completing a posterior arcade with a left superior pancreaticoduodenal artery. According to this report the terminal division of the gastroduodenal artery formed the right gastroepiploic and the right inferior pancreaticoduodenal arteries, the latter completing a second and lower posterior arcade with the left inferior pancreaticoduodenal artery from the superior mesenteric system. In this description emphasis is placed on the right and left relationships of the source vessels of the arcades and on the fact that both arcades are completed on the posterior aspect of the pancreas, one being higher or more eephalic than the other. Testut (1893) recognized two arches which he designated "peripancreatic arterial circles" but his more notable 
contribution is the designation of an inferior pancreatic artery found running along the dorsoinferior border of the body and tail of the pancreas. Testut described this as a branch of the superior mesenteric artery, a source which is probably a variation according to present day interpretations.

Do Rio-Branco ('12) presented a description of the major arteries of the pancreas, calling particular attention to the spiral course of the posterior superior pancreaticoduodenal artery in relation to the common bile duct. Many authors have since emphasized this close relationship. Romodanowskaja ('26) described the arteries of the pancreas on the basis of 34 dissections.

Although investigating only the vessels of the duodenum and head of the pancreas Petrén ('29) advanced considerably our knowledge of the arcades and their contributing arteries. He dissected 42 specimens and his cases are beautifully illustrated. This work marks the beginning of the modern terminology as to panereatic and duodenal branches of the gastroduodenal and superior mesenteric systems for Petrén showed that there was a greater difference in vertical than in horizontal separation of the vessels supplying the arches, especially so in the posterior arch. As a result later investigators have tended to follow Petrén in designating posterior superior and anterior superior pancreaticoduodenal branches of the gastroduodenal artery rather than right superior and right inferior branches, and posterior inferior and anterior inferior pancreaticoduodenal arteries from the superior mesenteric system rather than the respectively comparable left superior and left inferior pancreaticoduodenal arter:es.

This brief review of pertinent investigations may be concluded by reference to the study of Pierson ('43) who reported a careful series of dissections on 50 antopsy specimens and to that of Falconer and Griffiths ('50) whose investigation concerned both dissection and injection-corrosion material. Other investigators will receive mention as their studies become pertinent in relation to specific details hereafter. 


\section{PRESENTATION OF MATERIAL}

The following description of the pattern of arterial blood supply to the pancreas and the statistical analysis of variants in this pattern is based on detailed dissection by the authors of 150 specimens. A preliminary survey was made of a considerable number of specimens after dissection by students but it was found that destruction and loss of some vessels together with incomplete dissection of others made observations on this material so uncertain as to be worthless. Particularly was it true that vessels of apparently a certain source had a different origin than first appeared after a critical dissection was made. The material finally used was consecutive cadavers placed in the anatomical laboratories over several years' time but the authors made the dissection of the blood vessels in advance of student dissection in the region. Sketches of each specimen were prepared and the sketches were augmented by notations of origins and particular features.

\section{General pattern}

The authors would like to stress that there is a general pattern of vasculature here (fig. 1) which is remarkably constant, much more constant than previous studigs have indicated. There are two arterial arcades in relation to the head of the pancreas, one anterior and the other posterior; both serve to supply vasa recta to the duodenum and branches to the head of the pancreas. The anterior arcade occurred in every case in our series. The posterior arcade was lacking in only two cases, in one due to the absence of the posterior superior pancreaticoduodenal artery, in the other case due to the absence of the posterior inferior vessel.

There proved to be a constant terminal division of the gastroduodenal artery into right gastroepiploic and anterior superior pancreaticoduodenal arteries. The latter vessel descends on the anterior surface of the head of the pancreas, reaching the sulcus between the pancreas and duodenum at the junction of the second and third parts of the duodenum 
and there turns deeply to the dorsal aspect of the head of the pancreas. An open anastomosis of this vessel with the anterior inferior pancreaticoduodenal artery from the superios mesenteric system completes the anterior arcade. It will be apparent (fig. 1) that this inferior portion of the anterior arcade is formed on the dorsum of the pancreas in most cases, a circumstance which led Wiart (1899) to describe two

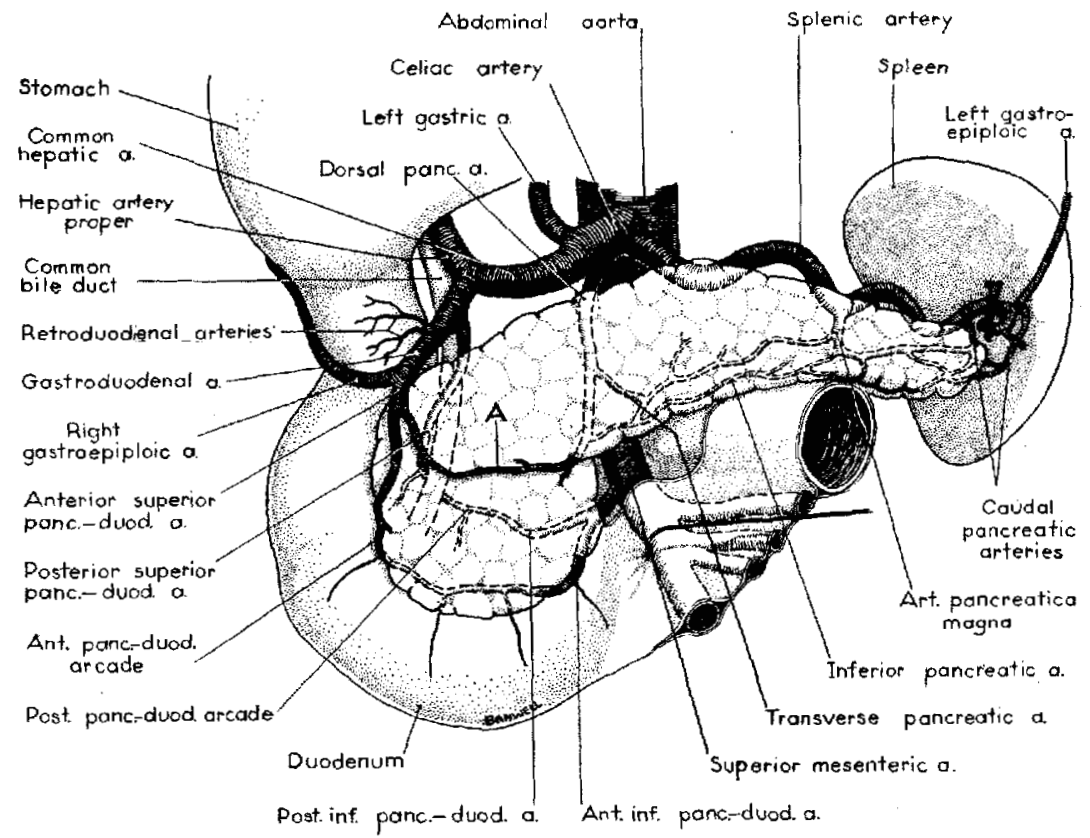

Fig. 1 General pattern of arterial supply of the pancreas. Symbol "A" designates a small but almost constant prepancreatic arterial arcade.

posterior arcades. However, the arcade is always present on the anterior surface of the pancreas in its superior portion and its vasa recta reach the anterior surface of the duodenum even in its inferior portion.

The posterior superior pancreaticoduodenal artery (fig. 1) is a proximal branch of the gastroduodenal artery arising from its retroduodenal portion. Although not mentioned in current American textbooks it has been recognized in the 
research literature since its first description by Haller in 1742 as the right superior pancreaticoduodenal artery. HalIcr's mention of this vessel and his description of two arcades of vessels appear to have been lost to the notice of his contemporaries and we find the next clear description of the arcades and their sources is that of Wiart in 1899. Verneuil (1851) clearly stated that two arcades existed but described both as formed from a descending branch of the hepatic and an ascending branch of the superior mesenteric artery. This inaccurate description delayed recognition of the important posterior superior pancreaticoduodenal artery and led ultimately to the investigations of do Rio-Branco ('12), Romodanowskaja ('26), and Petrén ('29). Do Rio-Branco ('12) emphasized that the artery in question (his right superior pancreaticoduodenal artery) arises $1-1.5 \mathrm{~cm}$ from the origin of the gastroduodenal artery as the latter reaches the superior border of the pancreas. This origin was to the left of the common bile duct in $42 \%$ of cases or along its left border in $38 \%$. It was to the right of the duct in only $20 \%$ of cases. In accordance with these findings do Rio-Branco described a first part of the artery running transversely to the right to cross the anterior face of the common bile duct at the level of the upper border of the pancreas. In its second segment the vessel turns the right flank of the common bile duct and sinks dorsally between pancreas and duodenum. Reaching a retropancreatic position it is directed inferiorly and to the left, crossing obliquely the posterior face of the retropancreatic common bile duct and anastomosing with the posterior inferior pancreaticoduodenal artery. The posterior superior pancreaticoduodenal artery has, contrary to the usage of all other authors, been designated the retroduodenal artery by Edwards ('41) and by Michels ('45 and '51). It is almost. constant in occurrence $(99.3 \%$ ) in our series and arises as the above described branch of the gastroduodenal artery in $92.6 \%$ of its total representation.

The foregoing discussion of the arcades and their sources has anticipated to a certain extent the deseription of the in- 
ferior pancreaticoduodenal arteries. The arcades are completed by open anastomoses with arteries arising from the superior mesenteric system, the detail of such origin showing considerable variability. Figure 1 displays the most common arrangement whereby the two inferior pancreaticoduodenal arteries are the products of division of a common stem arising from the superior mesenteric artery. This pattern accounts for $46 \%$ of the total representation. The source of these vessels is usually dorsal to the uncinate process or the neck of the pancreas and both are restricted in most cases to the dorsal aspect of the pancreatic head. Although the anterior inferior pancreaticoduodenal artery sends branches to the anterior surface of the third and 4th parts of the duodenum, it lies posterior to the uncinate process and the lower part of the head of the pancreas, becoming superficial to the pancreas only in the region of its anastomosis with the anterior superior pancreaticoduodenal artery. It has a $100 \%$ occurrence in our series of cases. The posterior inferior pancreaticoduodenal artery crosses the dorsum of the head of the pancreas more cephalically than the anterior inferior vessel and anastomoses with the posterior superior artery just medial to the common bile duct. The posterior inferior pancreaticoduodenal artery occurred in $99.3 \%$ of cases in our study, being absent only once. Duplication of these arcades is an occasional finding in dissection, occurring with greater frequency in the posterior areade. The frequency of such duplication is considerably greater if one counts the smaller intrapancreatic anastomoses.

The dorsal pancreatic artery (fig. 1) is a major artery of the pancreas which is not recognized in current texts and concerning which there is no little confusion in the research literature. It is the vessel which Haller designated as the great superior pancreatic artery and many authors have merely called the superior pancreatic artery. Since, however, there are a number of small superior pancreatic branches of the splenic artery and because of confusion with the currently recognized pancreatica magna artery it is deemed advisable 
to adopt the name of dorsal pancratic artery, from Michels' description. Do Rio-Branco used the term middle pancreatic artery. This vessel has a $90 \%$ occurrence in our series of dissections. It has traditionally been defined as a branch of the celiac, hepatic, or splenic artery arising within $1.5-2 \mathrm{~cm}$ of the origin of such vessel. It became apparent in our dissections that exactly the same artery with the same course and branches frequently arose from the superior mesenteric artery. Not all authors have included the latter source which accounts for considerable difference in the statistics of occurrence. The dorsal pancreatic artery descends dorsal to the neck or body of the pancreas, sometimes also being dorsal to the splenic vein, and toward the lower border of the pancreas divides into a right and a left branch. The right branch emerges on the surface of the pancreas at or near the junction of the uncinate process and the neck of the pancreas, sends a branch of supply down over the uncinate process and then turns across the head of the pancreas to make anastomosis with a small left branch of the anterior superior pancreaticoduodenal artery. This anastomosis forms a prepancreatic arterial arcade which supplies only pancreatic tissue and is labelled "A" in figure 1. It has a $93.3 \%$ occurrence in our series. In completing this anastomosis the left branch of the anterior superior pancreaticoduodenal may be substituted for by a branch of the adjacent right gastroepiploic artery or of the more proximal gastroduodenal artery. The left branch of the dorsal pancreatic vessel forms the inferior pancreatic artery next to be described.

The inferior pancreatic artery (fig, 1 ) is a vessel which runs along the inferior border of the body of the pancreas, frequently embedded in the dorsal aspect of that border and only partially visible from the surface of the pancreas. It is usually a continuation along the inferior edge of the pancreas of the left branch of the dorsal pancreatic artery. The name was first applied by Testut (1893) who described it as a branch of the superior mesenteric artery, presumably the left branch of a dorsal pancreatic artery from the superior mesenteric, 
as it would be analyzed under current concepts. The inferior pancreatic artery was designated the transverse pancreatic by Haller (1764). It usually anastomoses with candal pancreatic arteries and with radicles of the pancreatica magna artery and may communicate on the head of the gland with the prepancreatic arcade. It was a constantly occurring vessel in our series of dissections.

The pancreatica magna artery is described in current anatomy textbooks, usually as a large superior pancreatic branch of the splenic artery which enters the gland at approximately the junetion of the middle and left thirds of the gland. It penetrates immediately into the gland, sends branches to right and left which may be oriented along the main pancreatic duct, and anastomoses with other vessels within the pancreas. It had a $64.7 \%$ occurrence in our dissections. The earliest description of this artery available to the present writers is that in Quain's Elements of Anatomy of 1848 in which the vessel, after entering the gland, is described as passing from left to right along the main pancreatic duct.

Caudal pancreatic branches are to be found in most cases penetrating the tail of the pancreas to supply it and to form anastomoses with inferior pancreatic and pancreatica magna radicles. These vessels arise from the splenic artery before its division or from products of its division, notably the left gastroepiploic artery. Caudal pancreatic branches from these various sources occurred in $78.7 \%$ of our dissected specimens. They have been noted previously by many workers, especially Winslow (1732), Haller (1764), Pigache and Worms ('09), and Michels ('42).

\section{Variability of vessels}

The very considerable regularity of the general pattern of the arterial supply to the pancreas is evidenced in the high percentage occurrence of the vessels described heretofore. It will be of additional interest to examine the degree of variability in origin that these vessels exhibit. 
The origin and relations of the anterior superior pancreaticoduodenal artery are amazingly constant. Representing one of the terminal branches of the gastroduodenal artery, it had a 100\% occurrence in our series and there were no variations to report. Such has been the experience of almost all previous investigations also.

The variability of the posterior superior pancreaticoduodenal artery is also of minor degree as is evidenced by examination of figure 2 . Our $99.3 \%$ occurrence is only slightly less

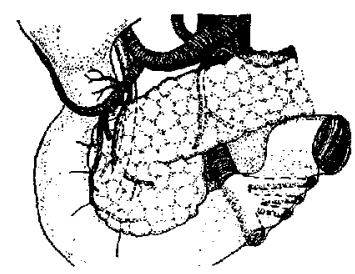

POST. SUP. PANCDUOD. A. FROM GASTRODUODEN. $-92.6 \%$

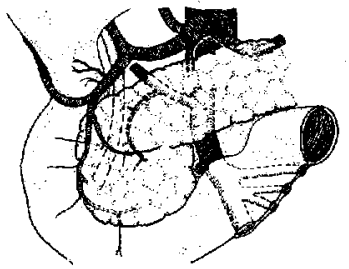

P. S. PANC.DUOD. FROM R, HEP. BR, OF SUP. MES, A.-3.4\%

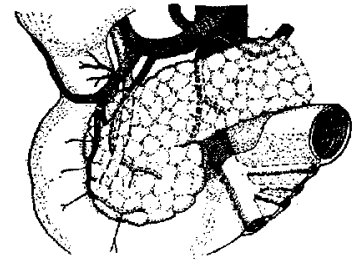

POST, SUP. PANC.DUOD. A. FROM HEPATIC A.-2.7\%

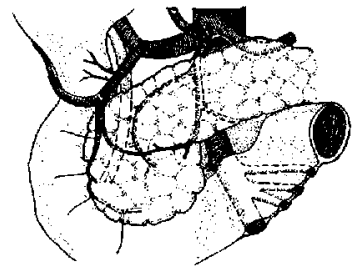

POST. SUP. PANC. DUOD. A. FROM DORS. PANC. A. $-1.3 \%$

Fig. 2 Varieties of superior panereaticoduodenal arteries. Anterior superior pane.duod, a. - occurrence $100 \%$ - variations none. Posterior superior pane.duod. a. - occurrence $99.3 \%$.

than Petrén's 100\% and but little greater than Edwards' $97 \%$ and Pierson's 96\%. All investigators have found a very high incidence of origin from the gastroduodenal artery and our $92.6 \%$ is virtually identical with Falconer and Griffiths' $92 \%$. Others, too, have found the next most frequent origin to be the accessory right hepatic branch of the superior mesenteric, $3 \%$ in the studies of both Petrén and Edwards. Reference has been made to the close relation of the posterior superior pancreaticoduodenal artery to the common bile duct. Do Rio-Branco found the artery arising to the left or in front of 
the duct in $80 \%$ and to its right in only $20 \%$. The comparable figures of Petrén were 86 and 14. Falconer and Griffiths found the artery "invariably in front of the common bile duct." Our investigations indicate that such was the case except when the origin of the vessel was bohind the head of the pancreas, as from the accessory right hepatic branch of the superior mesenteric or from the dorsal pancreatic artery.

Figure 3 illustrates the variability encountered in dissections of the inferior pancreaticoduodenal arteries. The reports
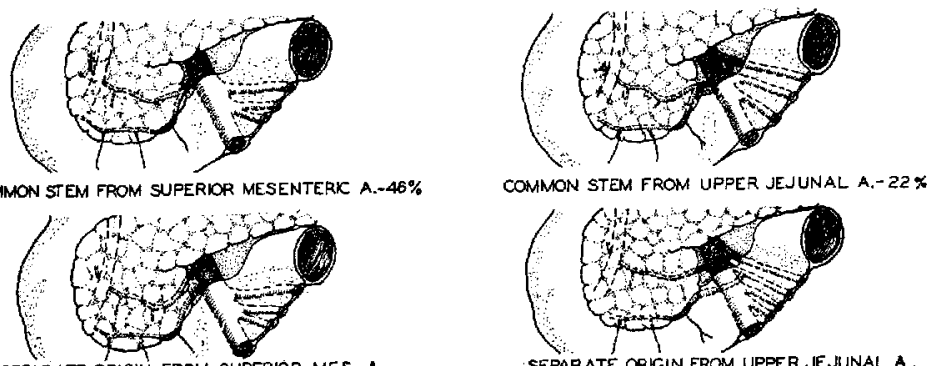

SEPARATE ORIGIN FROM SUPERIOR MES. A.

SEPAFATE ORIGIN FROM UPPER JEJUNAL A

POST. INF. PANC,DUOD.-15.5\%-ANT, INF. PANC.DUOD.- $16 \%$ POST, INF. PANC.DUOD. A.-7.4\%-ANT. INF. PANCDUOD. A.14,6\%
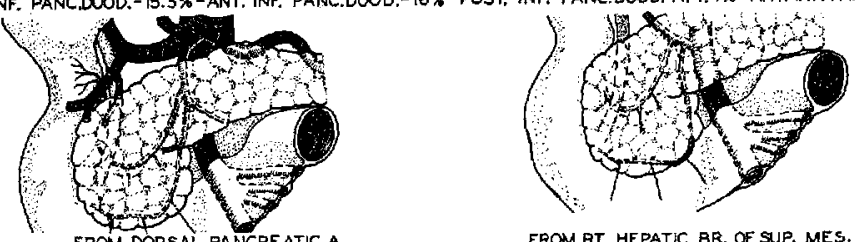

FROM RT HEPATIC BR OF SUP, MES. A.

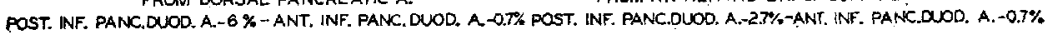

Fig. 3 Varieties of inferior pancreaticoduodenal arteries. Anterior inferior panc.duod. a.- oecurrence 100\%. Posterior inferior panc.duod. a.-occurrence $99.3 \%$.

of other authors bear out our results on general incidence and that these vessels most commonly arise by a common stem from either the superior mesenteric artery or one of its upper jejunal branches. Thus Shapiro and Robillard ('46) noted that a common stem was present in $70 \%$ of cases and assigned the upper jejunal origin for it in $20 \%$. It will be noted in figure 3 that either as separate origins or by a common stem the superior mesenteric system provides almost $99 \%$ of anterior inferior pancreaticoduodenal arteries and 
almost $91 \%$ of posterior inferior panereaticoduodenal vessels. It has been emphasized that both inferior pancreaticoduodenal arteries lie dorsal to the head of the pancreas or uncinate process. It should also be noted that they pass behind the superior mesenteric artery when arising from the upper jejunal arteries and (not illustrated) may take origin from the left side of the superior mesenteric or upper jejunal arteries.

The dorsal pancreatic artery (pancreatica magna suprema of Haller and superior pancreatic artery of certain authors) typically arises close to the aortic source of either the celiac or superior mesenteric systems. Its total occurrence has been variously reported as low as 50\% (do Rio-Branco) and as high as $90 \%$ (this investigation, fig. 4). Romodanowskaja ('26) found an occurrence of $56 \%$ in 34 specimens following the traditional definition of the artery as an early branch of the celiac, hepatic, or splenic artery. He observed, however, that the inferior pancreatic artery from the superior mesenteric alternated in incidence with such a dorsal pancreatic and had the same type of branches. The fact that the inferior pancreatic artery is usually the left branch of the dorsal pancreatic and that the latter does sometimes arise from the superior mesenteric gives a logical explanation to this observation. Adding Romodanowskaja's 11 cases of this type to his dorsal pancreatic incidence brings to $85 \%$ the total occurrence of this artery in his series. Falconer and Griffiths ('50) noted a $79 \%$ occurrence when all sources are included. The distribution of sources within the total varies in the reports of different observers. Do Rio-Branco assigned $50 \%$ to the splenic origin whereas Romodanowskaja found $50 \%$ from the celiac artery. Most earlier reports are somewhat unreliable because of the small number of specimens dissected and, with respect to adequate numbers, perhaps the present investigation (fig. 4) will yield truer figures of incidence from the various source vessels. It is probable, however, that those numerous vessels arising close to the division of the celiac artery pose 
problems of interpretation that will militate against complete agreement among investigators.

The right branch of the dorsal pancreatic artery extends across the head of the pancreas and forms a prepancreatic arcade by means of an anastomosis with a left branch of the anterior superior pancreaticoduodenal artery or an immediately related vessel. Do Rio-Branco ('12) stated that this arcade is almost constant, Pierson ('43) that it is fairly constant. Falconer and Griffiths ('50) found it in 11 of 27 cases.
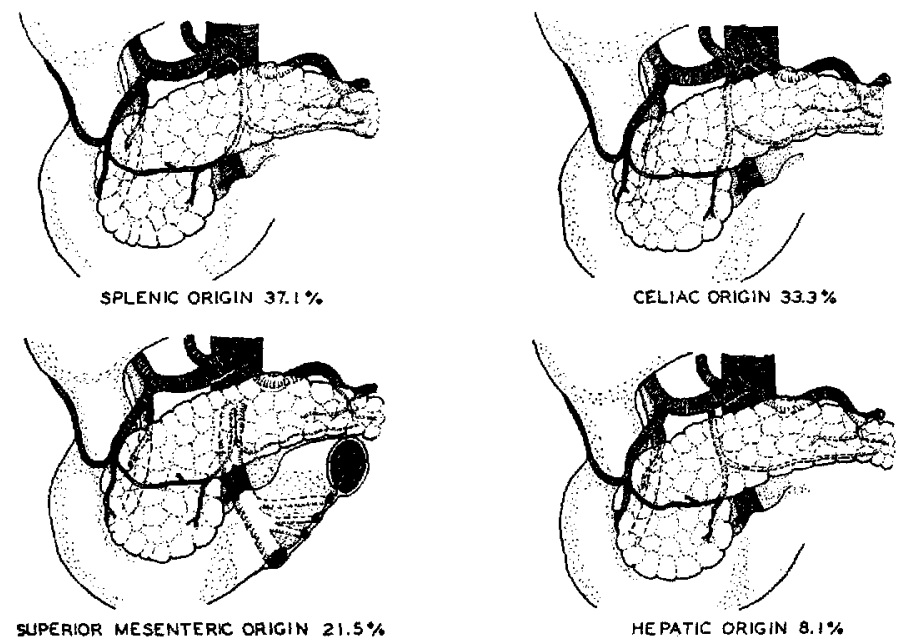

Fig. 4 Varieties of dorsal pancreatic artery. Oceurrence $90 \%$.

Romodanowskaja ('26) designated it as the anterior arcade (the pancreaticoduodenal arcades being considered as posterior) and treated it as a regularly occurring structure. The occurrence of this arcade in our specimens was $93.3 \%$. The middle colic artery is occasionally intimately related to the dorsal pancreatic artery. In our series the middle colic or an accessory vessel took origin from this pancreatic artery in $5 \%$ of the cases dissected.

The inferior pancreatic artery (fig. 5) had a $100 \%$ occurrence in our series of 150 dissections. Falconer and Griffiths ('50) found it in $89 \%$ in their series, while do Rio-Branco 
('12) merely gave it the designation of almost constant. Its predominant source is the left branch of the dorsal pancreatic artery which accounted for $84 \%$ of our specimens. When the dorsal pancreatic artery was missing the inferior pancreatic artery usually existed as a continuation of the left branch of the anterior superior pancreaticoduodenal artery; this occurred in $10 \%$ of our cases. Other minor sources for this vessel are listed in figure 5. We encountered the vessel as a separate branch of the superior mesenteric artery only rarely $(1.3 \%)$ although it was this source which Testut (1893) listed in his early description and Falconer and Griffiths ('50) cite this source for $37 \%$ of their specimens.

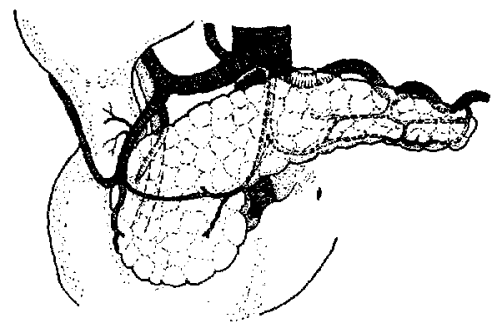

LEFT BR. OF DORSAL PANCRE ATIC A. $-84 \%$

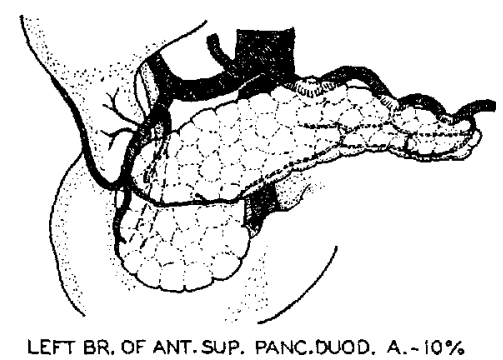

LEFT BR, OF ANT. SUP. PANC.DUOD. A. - 10\%

MINOR SOURCES - BR. OF GAST RODUODENAL A. $-2 \%$; SR. OF SUP. MES. A. $-1.3 \%$ BR. OF DORS. PANCR. +SUP. MES. A. $-1.3 \%$; BR. OF PANCRE ATIC A MAGNA A. $-1.3 \%$

Fig. 5 Varieties of inferior pancreatic artery. Occurrence $100 \%$.

Variability in the pancreatica magna artery exists in its occurrence (64.7\% in our series) and also in its exact position, but to be so designated it should be a larger superior pancreatic branch of the splenic artery entering the panereas at approximately the junction of its middle and left thirds. A vessel in this position and frequently oriented along the duct system has been described for over a century (Quain, 1848). The term designating the artery is open to question and may have led to confusion previously since Sobotta, writing in Bardeleben's Handbuch der Anatomie des Menschen ('14), stated that the arteria pancreatica magna of Haller is the first 
pancreatic branch of the splenic artery and is usually the larger of such branches. He obviously refers to the vessel designated magna suprema by Haller (here called the dorsal pancreatic artery).

\section{SUMMARY}

This study of 150 dissections indicates that:

1. There is considerable regularity in the pattern of arterial blood supply to the pancreas.

2. Two arterial arcades supply the duodenum and head of the pancreas.

3. The anterior arcade is formed by the constant anterior superior pancreaticoduodenal artery from the gastroduodenal artery anastomosing with the almost constant anterior inferior pancreaticoduodenal artery from the superior mesenteric system.

4. The posterior arcade is contributed to from above by the posterior superior pancreaticoduodenal artery, a proximal branch of the gastroduodenal artery. This arcade is completed by an almost constantly occurring posterior inferior pancreaticoduodenal artery arising in over 90\% of cases from the superior mesenteric system.

5. The inferior pancreaticoduodenal vessels are much more variable in the detail of their origin than the superior vessels.

6. A dorsal pancreatic artery occurs in $90 \%$ of cases. It exhibits a typical course and pattern of branches but arises variously from the splenic, the celiac, the superior mesenteric, or the hepatic artery. Its right branch crosses the head of the pancreas to form a prepancreatic arterial arcade (incidence $93.3 \%$ ) by means of an anastomosis with the left branch of the anterior superior pancreaticoduodenal artery.

7. The constant inferior pancreatic artery is found along the dorsoinferior border of the pancreas. It is in the majority of cases $(84 \%)$ the left branch of the dorsal pancreatic artery.

8. The pancreatica magna artery is a superior pancreatic branch of the splenic artery entering the body of the pancreas at the junction of its middle and left thirds and has an occurrence of $64.7 \%$. 
9. Caudal pancreatic arteries reach the tail from predominantly the splenic artery or its left gastroepiploic branch and occur in $78.7 \%$ of cases in this series.

\section{REFERENCES}

Do Rio-Brajico, P. 1912 Essai sur l’Anatomie et la Médecine opératoire du Tronc Coeliaque et de ses branches de l'Artère Hépatique en particulier. Thèse pour le Doctorat in Médecine; Faculté de Médecine de Paris.

EDWards, L. F. 1941 The retroduodenal artery. Anat. Ree., 81: 351-355.

FALCONGR, C. W. A., AND E. GRIFriths 1950 The anatomy of the blood vessels in the region of the pancreas. Brit. J. Surg., $37: 334-344$.

FALLER, A. 1742 H. Boerhaave praelectiones acad., 1: Taurini. 1764 Elementa Physiologiae Corporis Humani. VI, Lib. XXII.

Mтонви, N. 1942 The variational anatomy of the spleen and splenic artery. Am. J. Anat., 70: 21-72.

1945 Variations in blood supply of liver, gallbladder, stomach, duodenum and pancreas. J. Internat. Coll. Surg., 8: 502-504.

1951 The hepatic, eystic and retroduodenal arteries and their relations to the biliary ducts. Ann. Surg., 133: 503-524.

Petré, T. 1929 Die Arterien und Venen des Duodenums und des Pancreaskopfes beim Mensehen. Zeitsch. f. Anat. u. Entwicklungsgesch., 90: 234-277.

Pinesox, J. M. 1943 The arterial blood supply of the pancreas. Surg., Gyn., and Obs., $7 \%: 426-432$.

Pigache, R., and G. Worms 1909 Topographie du pédicule de la rate. Bull. et Mem. de Ia Soc. Anat. de Paris. Ser. 6, 11: 589-605.

QUaiN 1848 Elements of anatomy. v. 1, ed. by Richard Quain and William Sharpey. London, Walton and Maberly.

RomodanowskajA, Z. 1926 Die Arterien der Bauchspeicheldrüse. Zeitsch. $f$. Anat. u. Entwicklungsgesch., $79: 506-514$.

Shapiro, A. L., AND G. L. Robillakd 1946 Morphology and variations of the duodenal vasculature. Arch. Surg., 52: 571-602.

Sовотта, J. 1914 Anatomie der Bauchspeicheldrüse. Handb. der Anat. des Mensch. Ed. by Karl von Bardeleben. 6 (3) : 1-62.

Testup, L. 1893 Traité d'anatomie humaine. 3: 675-676. Paris, O. Doin. VkrnetIL, A. 1851 Mémoire sur quelques points de l'anatomie du pancréas. Gaz. Méd. de Paris. pp. 398-401.

WraRT, P. 1899 Recherches sur la forme et les rapports du panereas. J. de l'Anat. et de la Physiol., $35: 91-113$.

WINsLOW, J. B. 1732 An anatomical exposition of the structure of the human body. Translated from the French original by G. Douglas. London, Bettesworth, Hiteh, Osborn, Longman, Ware, Birt, Davis and Astley. 